\title{
Order effects in transitive inference: does the presentation order of social information affect transitive inference in social animals?
}

\section{OPEN ACCESS}

Edited by:

James A. R. Marshall,

University of Sheffield, UK

Reviewed by:

Pete C. Trimmer,

University of Bristol, UK

Marco Vasconcelos,

University of Minho, Portugal

${ }^{*}$ Correspondence:

Takashi Hotta,

Department of Biology and

Geosciences, Graduate School of

Science, Osaka City University,

Sumiyoshi, Sugimoto 3-3-138, Osaka,

558-8585, Japan

takasi712000@yahoo.co.jp

${ }^{\dagger}$ Present Address:

Lyndon A. Jordan,

Integrative Biology, The University of

Texas at Austin, USA;

Tomohiro Takeyama,

Department of Biosphere-Geosphere

Science, Faculty of

Biosphere-Geosphere Science,

Okayama University of Science,

Okayama, Japan

Specialty section

This article was submitted to Behavioral and Evolutionary Ecology,

a section of the journal

Frontiers in Ecology and Evolution

Received: 16 March 2015 Accepted: 28 May 2015

Published: 09 June 2015

Citation:

Hotta T, Jordan LA, Takeyama T and

Kohda M (2015) Order effects in

transitive inference: does the

presentation order of social

information affect transitive inference

in social animals?

Front. Ecol. Evol. 3:59.

doi: 10.3389/fevo.2015.00059

\section{Takashi Hotta *, Lyndon A. Jordan ${ }^{\dagger}$, Tomohiro Takeyama ${ }^{\dagger}$ and Masanori Kohda \\ Animal Society, Department of Biology and Geosciences, Graduate School of Science, Osaka City University, Osaka, Japan}

Transitive inference $(\mathrm{TI})$ is the ability to infer social relationships between individuals (e.g., if $A<B$ and $B<C$, then $A<C$ ), and has been documented in a variety of vertebrates. Many studies of TI use the task of inferring social dominance, where a subject animal $A$ first directly interacts with $B$ (e.g., $A$ subordinate to $B$ : $A<B$ ), and then indirectly observes the interaction of $B$ and an unknown $C(B<C)$, using both direct and indirect information to infer its own relationship with $C$ (i.e., $A<C$ ). However, order effects are known to influence learning, especially in complex scenarios, and we have little understanding of the effects of presentation order in transitive inference. Here we show that the cichlid Julidochromis transcriptus can use TI to correctly assess social relationships when information is presented in the order opposite to that most commonly employed in studies of TI. We find that focal individuals (A) can transitively infer their relationships with an unknown individual $(C)$ when initially given indirect experience (i.e., eavesdropping that $\mathrm{B}<\mathrm{C}$ ) and then given direct experience $(\mathrm{A}<\mathrm{B})$. We conclude that $J$. transcriptus can infer social relationships when experiencing first indirect and then direct social information. We suggest that in this and many other species, transitive inference may occur in either presentation order, and future studies of $\mathrm{TI}$ should account for order effects of social information.

Keywords: transitive inference, cooperatively breeding cichlid, information order, contest ability, dominance hierarchy

\section{Introduction}

Transitive inference (TI) is the ability to infer unknown relationships between objects by using multiple sources of information (Vasconcelos, 2008). For example, knowing that $\mathrm{A}<\mathrm{B}$ and $\mathrm{B}<\mathrm{C}$, the subject may infer that $\mathrm{A}<\mathrm{C}$. Although TI has long been considered a developmental milestone in human social and linguistic ability (Piaget, 1970), after the methodological development for animals (e.g., Bryant and Trabasso, 1971), a number of studies revealed that a variety of vertebrate social animals can also transitively infer social relationships (e.g., chimpanzee: Gillian, 1981; monkey: D'Amato and Columbo, 1988, 1990; lemur: MacLean et al., 2008; Tromp et al., 2014; rat: Daves, 1992; Birds: von Fersen et al., 1991; Bond et al., 2003; Weißet al., 2010; Mikolash et al., 2013; Fish: Grosenick et al., 2007).

Many TI studies document that highly social animals can infer the contest ability of unknown individuals based on information from direct experience and from social eavesdropping 
(e.g., Paz-y-Miño et al., 2004; MacLean et al., 2008; White and Gowan, 2013; Tromp et al., 2014) or operant conditioning (e.g., Gillian, 1981; von Fersen et al., 1991; Daves, 1992). Theoretical models predict that increased cognitive ability offers an adaptive advantage and evolves more easily in animals living in complex social groups (Byrne and Whiten, 1989). By inferring an unknown individual's relative dominance rank, animals may avoid costs of direct fights, including time and energy expenditure and risk of injury or predation. Thus, TI likely plays an important role in social rank estimation and the establishment or maintenance of dominance hierarchies (Cheney and Seyfarth, 1986; Hogue et al., 1996). In large and stable social groups, individuals frequently interact with many other group members within the communication network (McGregor and Peake, 2000). High cognitive abilities such as TI will be favored in these social groups where frequent interactions may otherwise lead to increased aggressive interactions.

Previous studies of TI demonstrate that subjects that directly fight with the known animal first, and are then allowed to observe aggressive interactions between known and unknown conspecifics, can infer dominance relationships of the unknown animal. Surprisingly, in most experimental studies of TI (Grosenick et al., 2007 being an exception for using only indirect information), the subject animal obtains information from direct fights with animal $\mathrm{B}$ first $(\mathrm{A}<\mathrm{B})$, and then gets indirect information from eavesdropping on the contest between $B$ and an unknown $\mathrm{C}(\mathrm{B}<\mathrm{C})$. Using this protocol, individual $\mathrm{A}$ has been shown to correctly infer the relative contest ability of animal C (i.e., A < C) in multiple species (e.g., Hogue et al., 1996; Pazy-Miño et al., 2004; Weißet al., 2010; Mikolash et al., 2013). But in natural social conditions, it is more likely that an incoming individual (A) will try to join a social group that has an already established dominance hierarchy (Jordan et al., 2010a,b). Indeed, in many species individuals visit groups many times before finally attempting to join them. This gives a potential joiner (A) the opportunity to observe the interactions of unknown members of the group before directly interacting with them (Vasconcelos, 2008).

More generally, in the field of learning and behavior, the effect of presentation order and serial learning are well known to influence retention of information (Domjan, 2010). It cannot be assumed for instance that a series of stimuli will be remembered in the same way presented in a different order (Domjan, 2010). In fact, researches of TI using operant conditioning tasks considered information presentation order (Steirn et al., 1995). In the context of social learning, indirect observations (i.e., that two unknown individuals have a certain dominance relationship, $\mathrm{B}<\mathrm{C}$ ) may constitute a less salient source of information, and so be more difficult to recall when presented with a direct interaction $(\mathrm{A}<\mathrm{B})$. Remarkably however, there appear to have been no studies of TI in which social information is presented in this alternative order (observation first and direct interaction second). We therefore do not know whether transitive inference is possible under these potentially more common social conditions.

Julidochromis transcriptus is a species indigenous to Lake Tanganyika, Africa (Konings, 1996) and is a cooperatively breeding cichlid with a highly organized social system (Awata and Kohda, 2004; Awata et al., 2005; Heg and Bachar, 2006; Kohda et al., 2009), which may favor higher cognition. This fish can recall memories of social events after 5 days (Hotta et al., 2014). In the previous experiment, we used three unknown individuals ( $\mathrm{A}, \mathrm{B}$, and $\mathrm{C})$. At first, $\mathrm{A}$ lost a competitive interaction with $B$ (i.e., $A<B$ ) and then $A$ observed $C$ defeating $B(B<C)$. As expected, in the first encounter, $A$ behaved subordinately to $C$ $(A<C)$, suggesting that $A$ infers social dominance transitively. We also showed that eavesdropping does not directly affect the reaction of the observer $\mathrm{A}$ to the interacting fish $\mathrm{B}$ or $\mathrm{C}$ in the absence of direct interaction (e.g., Oliveira et al., 1998), nor did we observer evidence of winner/loser effects following direct interactions (e.g., Hsu et al., 2006; Hotta et al., 2014). Taken together, these results demonstrate that J. transcriptus has the ability to perform TI to infer the dominant rank of unknown individuals when direct information precedes indirect information.

The purpose of this study is to test whether this fish can perform TI in the complementary presentation: indirect (eavesdropping) followed by direct (contest). Because our previous study showed no evidence that fish use social information alone to estimate the competitive ability of unknown individuals (Hotta et al., 2014), it is possible that TI is impossible when presented with social information prior to direct contests. It is therefore essential to establish whether fish can infer their relative dominance rank when the information order of social eavesdropping and direct experience is switched.

\section{Materials and Methods}

\section{Subjects and Housing}

Experiments were conducted in our laboratory at Osaka City University. The cichlid J. transcriptus used in this study were laboratory-reared descendants of wild-caught fish from Lake Tanganyika, Africa. We used males (60.6-76.0 mm in total length, TL) that had been kept with females in stock tanks, either $30 \times 40 \times 60 \mathrm{~cm}$ glass tanks of 20 individuals or $45 \times 40 \times$ $180 \mathrm{~cm}$ glass tanks of 60 individuals. These stock tanks contained multiple shelters of flower pots, stones and tiles put on coral gravel bottom and water was aerated and filtered. The tanks were kept at $24-26^{\circ} \mathrm{C}$ at a $12: 12$-h light/dark cycle. Fish were fed with artificial flake food (Tetramin) twice a day.

Three days before the start of the experiments, fish were measured TL and each placed in $30 \times 17 \times 15 \mathrm{~cm}$ glass tanks (house tank) with $2 \mathrm{~cm}$ coral substrate, filtration and aeration. The fish were visually isolated from one another: all sides of the tank were covered with opaque sheets. This isolation lasted for 14 days prior to the experiment to avoid any effects of previous contests or social experience (Hsu et al., 2006; Hotta et al., 2014).

\section{Experimental Procedure}

We designed 3-term series tasks (Bryant and Trabasso, 1971) that have previously been used in studies of social transitive inference (Hogue et al., 1996; Paz-y-Miño et al., 2004; White and Gowan, 2013). We made 31 triads containing individuals were 
size-matched (TL difference was within $3 \mathrm{~mm}$, and preliminary experiments showed that the size difference was sufficiently small that we could not a priori predict the winner of the contest). This experiment consisted of three phases, a pre-phase I, a pre-phase II and a test phase.

In the pre-phase I, two fish were placed in an arena tank, and watched by an observer fish A that was physically separated from the arena tank for $30 \mathrm{~min}$. Our preliminary experiment showed that dominance relationships took up to $15 \mathrm{~min}$ to stabilize, i.e., one fish behaving aggressively (performing chases and bites) and another fish adopting submissive behaviors and retreating when another fish approaches. Thus we allowed $30 \mathrm{~min}$ to establish dominance after introducing two size-matched fish to arena tanks. The tank housing the observer fish A was separated by $7 \mathrm{~cm}$ space from the tank housing the physically interacting fish (B and C), and we did not observe any behavioral interactions among observing and interacting fish. After $30 \mathrm{~min}$, we measured the dominance relationship between the interacting fish, referring to the winner of the dyadic interaction as " $\mathrm{C}$," and the loser as " $\mathrm{B}$ " (Figure 1).

In the pre-phase II, the loser fish B and the previous observer fish A were transferred into a new arena tank, and the winner fish $\mathrm{C}$ was moved to a new observer tank to be allowed to observe interactions between $A$ and $B$ at a distance of $7 \mathrm{~cm}$. As in the pre-phase I, we observed the physical interaction during $30 \mathrm{~min}$ and determined dominance relationships. In this study, the effect of information order was evaluated by comparison with results from experiments using traditional social information presentation order (direct followed by indirect). The experience of winning and being observed did not influence contest behavior in J. transcriptus (Hotta et al., 2014). If fish B was dominant over fish $\mathrm{A}$, the loser fish $\mathrm{A}$ and the observer fish $\mathrm{C}$ were transferred to a paired test tank (Figure 1, $n=18$ ). Conversely, when the fish A was dominant over the fish $B$, the experiment was terminated and the fish were returned to own house tank $(n=13)$. At first, an opaque sheet was placed between the test tank and fish were acclimated for $10 \mathrm{~min}$. After the acclimation period, the opaque sheet was removed and behavior was recorded for $10 \mathrm{~min}$ by video camera (HDR-CX370, Sony). This procedure allowed visual, but not physical, interaction.

During the first $30 \mathrm{~s}$ of exposure in the test phase, we measured the following responses of each individual to evaluate aggressive behavior: "rushing time" (when the focal fish rapidly swims toward the glass barrier) and "time in near zone" (when the bottom of the tank was divided into three zones, i.e., each zone is $5.7 \mathrm{~cm}$, the time fish stayed in a zone near glass barrier). Subordinate behavior was defined as one fish showing shorter rushing time and less time in near zone than their opponent. These responses and the observation time were similar to previous studies (Hotta et al., 2014).

To create a directly comparable experimental design to previous studies of transitive inference, we allowed fish $\mathrm{C}$ to observe the contest between A and B, because traditional TI studies allow an audience to observe all contests. However, this has the effect of presenting social contest information to fish $\mathrm{C}$ in the traditional order, creating the potential for "normal" transitive inference on the part of C. Using normal TI, fish C may therefore infer that it is dominant over $\mathrm{A}$, and be more aggressive toward $\mathrm{A}$ in the final trials. This increased aggression may elicit a subordinate response in A that is unrelated to transitive inference. To test for this possibility, we compared the level of aggression shown by $\mathrm{C}$ to that shown in contests between two unfamiliar fish that were placed in test-phase contests but had no previous social interactions. The null expectation is that fish $\mathrm{C}$ will show levels of aggression that are not significantly different to those of unfamiliar fish, and consequently that any change in behavior in $\mathrm{A}$ is due to transitive inference rather than a reaction to increased aggression by $\mathrm{C}$. Note that this test does not eliminate the possibility that $\mathrm{C}$ is performing TI, but only that this does not result in increased aggression by C. Alternatively, if fish $\mathrm{C}$ shows higher than normal aggression, we cannot conclude that any change in the behavior of $\mathrm{A}$ is a consequence of TI.

All experiments were conducted in compliance with Regulations on Animal Experiments at Osaka City University and the Japanese Ethological Society. During physical contests in pre-phase I and pre-phase II, when fish had the opportunity to engage in open aggression, e.g., mouth wrestling, all contests were video-taped and monitored by an observer. If either fish appeared to suffer visible physical injury, the observer would have terminated the contests. However, we did not observe any fish suffering physical injury.

\section{Statistical Analyses}

Statistical analyses were conducted in $\mathrm{R}$ version 3.0.0. Wilcoxon signed-rank tests were used to compare rushing time and time in near zone between fish $\mathrm{A}$ and $\mathrm{C}$ during the test phase to determine whether fish A acted subordinately in the absence of direct experience with $\mathrm{C}$ (i.e., used transitive inference to infer that $\mathrm{C}$ is dominant over A). Additionally, Mann-Whitney $U$ tests were used to compare the traditional and reversed social information presentation order, in order to determine whether the order of social information presentation influences TI.

\section{Results}

In the test phase, rushing time by fish $\mathrm{A}$ against fish $\mathrm{C}$ was significantly shorter than fish $\mathrm{C}$ against fish $\mathrm{A}$ (Wilcoxon signedrank test: $T=290, N=18, p<0.005$; Figure 2A). Fish A stayed in the near zone for a significantly shorter total time than fish C (Wilcoxon test: $T=243, p<0.01$; Figure 2B). Thus, fish A behaved subordinately to fish C. In addition, the level of aggression shown by fish $\mathrm{C}$ was not different from that shown by unfamiliar fish that had no previous social interactions (MannWhitney test: $U=162, \mathrm{~N} 1=18, \mathrm{~N} 2=14$, in rushing time, $p=0.18 ; U=164, \mathrm{~N} 1=18, \mathrm{~N} 2=14$, in time in near zone, $p=0.15$ ).

Further, rushing time and time in the near zone of fish $\mathrm{A}$ in the present study (with reversed social information presentation order) was not significantly different from rushing time and time in the near zone of fish A in our previous study (with traditional social information presentation order; Mann-Whitney test: $U=$ $173, \mathrm{~N} 1=18, \mathrm{~N} 2=15$, in rushing time, $p=0.17 ; U=128$, $\mathrm{N} 1=18, \mathrm{~N} 2=15$, in time in near zone, $p=0.80$ ). 


FIGURE 1 | Procedure of experiments. In pre-phase I, A observes that C defeats B (B < C). Then A is defeated by B (A < B), and C observes that contest in
pre-phase II. Finally, in test-phase, $\mathrm{A}$ is faced to confront with $\mathrm{C}$ and their behaviors are video-recorded. (See text for detail).

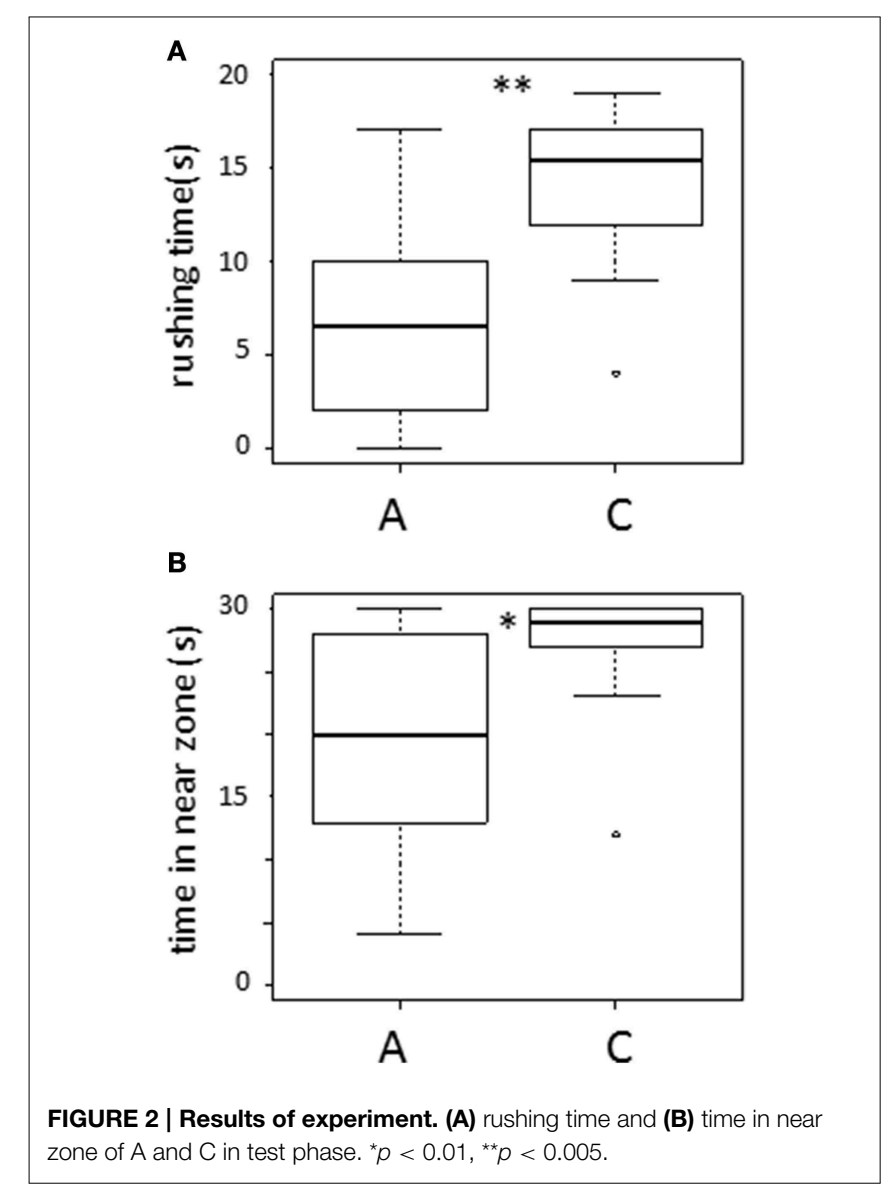

\section{Discussion}

In this study, we tested whether the male cichlids Julidochromis transcriptus use TI to infer the relative contest ability of an unknown individual by observing a physical contest between two unknown individuals first, and then directly interacting with the previous loser. This order of social experience has never before been tested in the context of transitive inference, with previous studies either beginning with direct exposure (contests) and following with indirect exposure (eavesdropping), or using only indirect social information (Grosenick et al., 2007). However, in learning studies, the order of presentation is known to have strong effects on learning and memory (Domjan, 2010). Moreover, indirect experiences followed by direct experiences are potentially more ecologically relevant and more common in group joining animals than direct followed by indirect experiences (Bergmuller et al., 2005; Jordan et al., 2010a,b). Our results showed that subject fish $(\mathrm{A})$ were able to transitively infer their social relationship with an unknown fish (C) when first observing $\mathrm{C}$ interact with $\mathrm{B}$, and then directly interacting with B. Focal fish behaved subordinately to $\mathrm{C}$ despite never having interacted directly with this previously unknown fish. There are two explanations for why fish A would behave subordinately to fish C. Firstly, the subordinate behavior of fish A may be a direct response to increased aggression from fish $\mathrm{C}$, which has used "traditional" transitive inference to determine it is dominant over fish A. However, we found that the aggressive behavior of fish $\mathrm{C}$ was not different from that of unfamiliar fish, and therefore does not explain the observed change in the behavior of A. It should be emphasized that this test does not confirm that fish $\mathrm{C}$ is not using TI in the traditional manner, only that it does not show any observable behavioral correlate of TI if it is being used. An alternative explanation for this pattern of behavior is that fish A is displaying a loser effect (Hsu et al., 2006) due to a recent contest loss (against B), and that fish $\mathrm{C}$ displays some form of winner effect (i.e., dominance badge, Colgan, 1983), or that social eavesdropping on the interaction between $B$ and $C$ is sufficient to change the behavior of A. However, our previous studies find no evidence that $J$. transcriptus exhibits dominance badges, loser effects, or social eavesdropping (Hotta et al., 2014).

The comparison between traditional and reversed social information presentation order shows that there are no differences in the social responses of fish A against winner C, suggesting that both orders of presentation are equally effective in inducing transitive inference. Furthermore, almost all individuals displayed TI under both order presentations (11/12 fish can do TI for $\mathrm{A}>\mathrm{C}$ in the previous study, and $17 / 18$ in the present study; $\chi^{2}$-test, $\chi^{2}=0.015, p=0.91$ ). Combined, our current and previous results demonstrate that a single source of social information is not sufficient to induce submissive behavior to an unknown individual, and that the combination of direct and indirect social experience is sufficient for TI, independent of presentation order.

A previous study on this species suggests that $J$. transcriptus do not use eavesdropping alone to determine dominance 
relationships (Hotta et al., 2014). However, we cannot judge whether observing fish are able to determine the social relationships by eavesdropping, but do not use this knowledge in their subsequent direct interactions, or if they are simply unable to assess dominance relationships through observation alone. In the present study, this fish eavesdrops on the interactions of two unknown fish first, and if it then loses in a direct contest with the observed loser, it transitively infers its relationship with the observed winner. This strongly suggests that these fish are able to assess and remember social relationships based on observation alone, but do not use this information in the absence of a direct interaction. This is in keeping with observations of other social animals that use TI but not social eavesdropping alone (e.g., hen: Hogue et al., 1996; pinyon jay: Paz-y-Miño et al., 2004), and we suggest these animals are also able to recognize and memorize the outcome of observed contests, but do not modify their behavior based on indirect experiences alone. It is possible that observed contests provide less reliable social information, and animals making judgements on indirect information alone may suffer increased costs of inappropriate social contests.

When attempting to join social groups of unknown individuals, individuals will most likely indirectly observe the interactions of some or most members before directly interacting with them. Prospecting behavior, in which an animal visits groups numerous times before eventually joining them (Bergmuller et al., 2005), would allow for numerous indirect observations of social interactions, allowing rapid assessment of dominance relationships using transitive inference when joining groups. Prior to this study, however, it was not known

\section{References}

Awata, S., and Kohda, M. (2004). Parental roles and the amount of care in a biparental substrate brooding cichlid: the effect of size differences within pairs. Behaviour 141, 1135-1149. doi: 10.1163/1568539042664623

Awata, S., Munehara, M., and Kohda, M. (2005). Social system and reproduction of helpers in a cooperatively breeding cichlid fish (Julidochromis ornatus) in Lake Tanganyika: field observations and parentage analyses. Behav. Ecol. Sociobiol. 58, 506-516. doi: 10.1007/s00265-005-0934-6

Bergmuller, R., Heg, D., Peer, K., and Taborsky, M. (2005). Extended safe havens and between group dispersal of helpers in a cooperatively breeding cichlid. Behaviour 142, 1643-1667. doi: 10.1163/156853905774831800

Bond, A. B., Kamil, A. C., and Balda, R. P. (2003). Social complexity and transitive inference in corvids. Anim. Behav. 65, 479-487. doi: 10.1006/anbe. 2003.2101

Bryant, P. E., and Trabasso, T. (1971). Transitive inferences and memory young children. Psycol. Bull. 89, 456-458.

Byrne, R. W., and Whiten, A. (1989). Machiavellian Intelligence: Social Expertise and the Evolution of Intellect in Monkeys, Apes, and Humans. London: Oxford Science Publications.

Cheney, D. L., and Seyfarth, R. M. (1986). The recognition of social relations by monkeys. Cognition 37, 67-196.

Colgan, P. (1983). Comparative Social Recognition. New York, NY: Wiley.

D'Amato, M. R., and Columbo, M. (1988). Representation of serial order in monkeys (Cebus apella). J. Exp. Psycol. 14, 131-139.

D'Amato, M. R., and Columbo, M. (1990). The symbolic distance effect in monkeys (Cebus paella). Anim. Learn. Behav. 18, 133-140. doi: 10.3758/BF03205250

Daves, H. (1992). Transitive inference in rats (Rattus norvegicus). J. Comp. Psychol. $106,342-349$. if animals could perform TI when presented indirect followed by direct social information. In our experiment, we show that fish are able to perform TI when presented with information in the order expected when joining new social groups. Further TI studies should be conducted across taxa focusing in the order of presentation of social information.

In conclusion, we show that $J$. transcriptus can transitively infer their social relationship with unknown individuals, and that the order of social information presentation (direct and indirect) does not influence the ability to perform TI. Our experimental procedures provide a straightforward method to test TI and other social abilities in fish (direct fighting, eavesdropping, winner/loser effects).

\section{Author Contributions}

MK and TT developed the study concept. TH, MK and TT contributed to the study design. Testing, date collection and the date analysis were performed by $\mathrm{TH}$. TH drafted the manuscript, and $\mathrm{AJ}$ and $\mathrm{MK}$ provided critical revisions. All authors approved the final version of manuscript for submission.

\section{Acknowledgments}

We are grateful to members of the animal society research group in Osaka City University for valuable discussion. This work was financially supported by the Grand-in-Aid from Ministry of Education, Science, Sports, Culture and Technology of Japanese Government to MK (Nos. 26118511 \& 26540070, and on Innovative Areas No. 4501) from the JSPS in Japan.
Domjan, M. (2010). The Principles of Learning and Behaviour, 6th Edn. Belmont: Cengage learning.

Gillian, D. J. (1981). Reasoning in chimpanzee II: transitive inference. J. Exp. Psycol. 7, 150-164.

Grosenick, L., Clement, T. S., and Fernald, R. D. (2007). Fish can infer social rank by observation alone. Nature 445, 429-432. doi: 10.1038/nature05511

Heg, D., and Bachar, Z. (2006). Cooperative breeding in Lake Tanganyika cichlid Julidochromis ornatus. Environ. Biol. Fish. 76, 265-281. doi: 10.1111/j.14390310.2005.01135.x

Hogue, M. E., Beaugrand, J. P., and Lague, P. C. (1996). Coherent use of information by hens observing their former dominant defeating or being defeated by a stranger. Behav. Proc. 38, 241-252.

Hotta, T., Takeyama, T., Jordan, L. A., and Kohda, M. (2014). Duration of memory of dominance relationships in a group living cichlid. Naturwissenschaften 101, 745-751. doi: 10.1007/s00114-014-1213-z

Hsu, Y., Earley, R. L., and Wolf, L. L. (2006). Modulation of aggressive behaviour by fighting experience: mechanisms and contest outcomes. Biol. Rev. 81, 33-74. doi: $10.1017 /$ S146479310500686X

Jordan, L. A., Avolio, C., Herbert-Read, J. E., Krause, J., Rubenstein, D., and Ward, A. (2010a). Group structure in a restricted entry system is mediated by both resident and joiner preferences. Behav. Ecol. Sociobiol. 64, 1099-1106. doi: 10.1007/s00265-010-0924-1

Jordan, L. A., Wong, M. Y. L., and Balshine, S. (2010b). The effects of familiarity and social hierarchy on group membership decisions in a social fish. Biol. Lett. 6, 301-303. doi: 10.1098/rsbl.2009.0732

Kohda, M., Heg, D., Makino, Y., Takeyama, T., Shibata, J., Watanabe, K., et al. (2009). Living on the wedge: female control of paternity in a cooperatively polyandrous cichlid. Proc. R. Soc. Lond. B. 276, 4207-4214. doi: 10.1098/rspb.2009.1175 
Konings, A. (1996). Guide to Tanganyika Cichlids. Ahornweg: Cichlid Press.

MacLean, E. L., Merritt, D. J., and Brannon, E. M. (2008). Social complexity predicts transitive reasoning in prosimian primates. Anim. Behav. 76, 177-182. doi: 10.1016/j.anbehav.2008.01.025

McGregor, P. K., and Peake, T. M. (2000). Communication networks: social environments for receiving and signaling behaviour. Acta. Ethol. 2, 71-81. doi: $10.1007 / \mathrm{s} 102110000015$

Mikolash, S., Kotrschal, K., and Schloegl, C. (2013). Transitive inference in jackdaws (Corvus monedula). Behav. Proc. 92, 113-117. doi: 10.1016/j.beproc.2012.10.017

Oliveira, R. F., McGregor, P. K., and Latruffe, C. (1998). Know thine enemy: fighting fish gather information from observing conspecific interactions. Proc. R. Soc. Lond. B. 276, 2249-2256. doi: 10.1098/rspb.2009.0132

Paz-y-Miño, C. G., Bond, A. B., Kamil, A. C., and Balda, R. P. (2004). Pinyon jays use transitive inference to predict social dominance. Nature 430, 778-781.

Piaget, J. (1970). Genetic Epistemology. New York, NY: Columbia University Press.

Steirn, J. N., Weaver, J. E., and Zentall, T. R. (1995). Transitive inference in pigeons: Simplified procedures and a test of value transfer theory. Anim. Learn. Behav. 23, 76-82.

Tromp, D., Meunier, H., and Roeder, J. J. (2014). Transitive inference in two lemur species (Eulemur macaco and Eulemur fulvus). Ame. J. Primatol. doi: 10.1002/ajp.22349
Vasconcelos, M. (2008). Transitive inference in non-human animals: an empirical and theoretical analysis. Behav. Proc. 78, 313-334. doi: 10.1016/j.beproc.2008.02.017

von Fersen, L., Wynne, C. D. L., and Delius, J. D. (1991). Transitive inference in pigeons. J. Exp. Psycol. 17, 334-341.

Weiß, B. M., Kehmeier, S., and Schloegl, C. (2010). Transitive inference in free-living graylag geese, Anser anser. Anim. Behav. 79, 1277-1283. doi: 10.1016/j.anbehav.2010.02.029

White, S. L., and Gowan, C. (2013). Brook trout use individual recognition and transitive inference to determine social rank. Behav. Ecol. 24, 63-69. doi: 10.1093/beheco/ars136

Conflict of Interest Statement: The authors declare that the research was conducted in the absence of any commercial or financial relationships that could be construed as a potential conflict of interest.

Copyright (c) 2015 Hotta, Jordan, Takeyama and Kohda. This is an open-access article distributed under the terms of the Creative Commons Attribution License (CC $B Y)$. The use, distribution or reproduction in other forums is permitted, provided the original author(s) or licensor are credited and that the original publication in this journal is cited, in accordance with accepted academic practice. No use, distribution or reproduction is permitted which does not comply with these terms. 